\title{
Evaluating voice traffic requirements on IEEE 802.11 ad hoc networks
}

\author{
Pedro Braconnot Velloso ${ }^{1}$, Marcelo G. Rubinstein ${ }^{2}$, Otto Carlos M. B. Duarte ${ }^{3}$ \\ 1 LIP6/CNRS - Université Pierre et Marie Curie \\ 8, rue du Capitaine Scott - 75015 - Paris - France \\ 2 PEL/DETEL-FEN - Universidade do Estado do Rio de Janeiro \\ R. São FCo. Xavier, 524 - 20550-013 - Rio de Janeiro - RJ - Brazil \\ 3 GTA/COPPE/POli \\ Universidade Federal do Rio de Janeiro \\ P.O. Box 68504 - 21945-970 - Rio de Janeiro - RJ - Brazil
}

\begin{abstract}
This paper analyzes voice transmission capacity on IEEE 802.11 ad hoc networks by performing simulations related to delay, jitter, loss rate, and consecutive losses. We evaluate the influence of mobility on the number of sources transmitting voice. Another issue addressed in this paper is the effect of node density on voice transmission. Our simulation model has allowed us to identify the main reasons for voice degradation in ad hoc networks. Results show that voice transmission capacity degrades with mobility and network load, being more sensitive to high mobility due to link failures. The network capacity can easily experience a decrease of up to 60\% on the number of voice transmissions on a multihop environment. We also show that node density is also relevant when considering voice transmission on multihop networks.
\end{abstract}

Keywords: Wireless ad hoc networks, IEEE 802.11, voice transmission, and mobility.

\section{Introduction}

Nowadays, wireless communication plays an important role in computer networks due to its high flexibility and low implementation cost. Although wireless local area networks are becoming common place, transmission of real-time traffic is still a great challenge.

Wireless communications can use infrastructure mode, where all communications take place through an access point or can operate on ad hoc mode, which is characterized by no infrastructure and by nodes that directly communicate with each other. The main advantages of ad hoc networks are flexibility, low cost, and robustness. Ad hoc networks can be easily set up, even in desert places, and can endure to natural catastrophes and war. Therefore, ad hoc networks fit well where there is no infrastructure and it is too expensive to build it, or when local infrastructure is not reliable, as for military operations in the enemy territory. Nevertheless, each node must implement distributed medium access control mechanisms and deal with exposed and hidden terminal problems. These mechanisms add considerable complexity to nodes, especially on multihop networks, where nodes also act as routers. Besides, ad hoc networks must cope with other wireless problems, such as low transmission rate, high Bit Error Rate (BER), and significant variations in physical medium conditions. This complexity makes transmission of real-time traffic on ad hoc networks a great challenge due to Quality of Service (QoS) requirements.

Some researchers have studied voice transmission on IEEE 802.11 networks on infrastructure mode. Köpsel et al. [1] analyze DCF (Distributed Coordination Function) and PCF (Point Coordination Function) mechanisms with respect to 
the number of nodes transmitting voice traffic and propose a hybrid mechanism using DCF and PCF modes. In order to improve network performance they also present an optimal switching point from DCF to PCF mode. Köpsel et al. also present in [2] an analysis of DCF and PCF considering the number of voice traffics and BER. Garg and Kappes [3] present delay and jitter results for voice traffic through experiments. Hole and Tobagi [4] perform similar analyses using simulation. All these previous researches consider the infrastructure mode of IEEE 802.11 networks. Concerning voice transmission on ad hoc networks, Deng and Chang [5] investigate through simulations the influence of DiffServ (Differentiated Services) on delay and loss probability for voice traffic in single hop ad hoc networks. However, results covering the multihop ad hoc mode have not been well explored yet. Armenia et al. [6] evaluate the performance of voice traffic on a multihop ad hoc network with only five static nodes. Experimental results related to throughput, delay, and jitter are presented.

To be best of our knowledge, there is no work that makes a throughout evaluation of voice traffic on multihop ad hoc networks. The main goal of our work is to analyze the capacity of voice transmission on multihop ad hoc networks and to evaluate the impact of mobility on voice traffic. This paper analyzes the effect of mobility on the capacity of voice transmission in ad hoc networks [7]. We also show the difference between the capacity of single hop networks and multihop networks [8]. Another issue addressed in this paper is the impact of network node density on the capacity of voice transmission. Therefore, we evaluate the behavior of Qos parameters, such as, loss rate, delay, jitter, and consecutive losses, under different network conditions.

The remainder of this paper is organized as follows. Section II briefly summarizes main QoS parameters related to real-time voice traffic. Simulation results are shown in section III. Section IV presents our conclusions.

\section{Voice transmission on IEEE 802.11 networks}

Real-time voice traffic has Qos requirements such as bounded end-to-end delay, maximum jitter, and limited loss rate. Different from data traffic, voice traffic supports a limited packet loss rate and is sensitive to the number of consecutive packet losses. Moreover, audio stream must be presented at the receiver with the same temporal relationship as it was captured. Therefore, jitter becomes an important QoS parameter strongly related to synchronization and, consequently, to buffering features at the receiver. End-to-end delay plays an important role in interactivity. This delay consists of four basic components: coding/decoding delay, packet generation delay, propagation delay, and queuing delay. Table I presents some reference values of tolerance to delay recommended by the ITU-T [9].

Table I: Tolerance to delay in voice communications.

\begin{tabular}{|c|c|}
\hline Delay (ms) & Tolerance \\
\hline less than 150 & good interactivity \\
\hline 150-400 & user can notice loss of interactivity \\
\hline over 400 & lack of interactivity \\
\hline
\end{tabular}

IEEE 802.11 [10] is the most widespread wireless network technology. It includes physical and link layer specifications. At the link layer, two MAC (Medium Access Control) methods are available. Distributed Coordination Function (DCF) is a basic mechanism that supports infrastructureless networks. A centralized mechanism called Point Coordination Function (PCF) supports real-time traffic, but demands an access point. 
DCF is a distributed mechanism based on CSMA/CA (Carrier sense Multiple Access with Collision Avoidance) in which every station must sense the medium before transmitting any frame. If the medium is idle the station must wait for DIFS (Distributed Inter-Frame Space) units of time. Then, if the medium is still idle, the sender should wait for a random time interval (backoff) between zero and the maximum contention window. By the end of the backoff time, the sender finally transmits. Backoff is part of the collision avoidance mechanism.

In wireless communications there is a great difference between received and transmitted signal power due to significant signal attenuation. Therefore wireless nodes are not capable of detecting collision at the recipient. In order to improve medium efficiency, a Cyclic Redundancy Check (CRC) with positive acknowledgment (ACK) is used for error control. If the frame seems to be correct, the recipient sends an ACK to the sender after sensing the medium idle for a period of time called SIFS (Short Inter-Frame Space). By definition, SIFS is smaller than DIFS.

The DCF method also optionally uses Request to send (RTS) and Clear to send (CTS) frames to avoid the hidden terminal problem [10].

Although multihop ad hoc networks are not the primary target of the IEEE 802.11 standard, several researchers have been using this technology with ad hoc routing protocols to deploy multihop ad hoc networks.

In this paper we have simulated voice transmission on a single hop IEEE 802.11 ad hoc network and then on a multihop IEEE 802.11 network in order to compare the capacity of these networks to carry voice, according to a maximum loss rate. We have considered end-to-end delay, jitter, packet loss rate, and consecutive losses in the analysis.

\section{Simulation Results}

This section describes the simulation model and presents the results obtained using the ns-2 network simulator [11]. For all simulations, the data rate at the physical layer is $11 \mathrm{Mbps}$ and the routing protocol is DSR (Dynamic Source Routing [12]), which is a well-known routing protocol for ad hoc networks. Main simulation parameters are presented in Table II. The results consider $90 \%$ confidence intervals for the mean that are represented in the figures by vertical bars.

Table II: Simulation parameters.

\begin{tabular}{|l|c|l|l|}
\hline Parameter & Value & Parameter & Value \\
\hline Transmission rate & $11 \mathrm{Mbps}$ & Header & 48 bits \\
\hline Slot time & $20 \mu \mathrm{s}$ & $\mathrm{CW}_{\min }$ & 31 slots \\
\hline SIFS & $10 \mu \mathrm{s}$ & $\mathrm{CW}_{\max }$ & 1023 slots \\
\hline DIFS & $50 \mu \mathrm{s}$ & ACK frame size & 14 bytes \\
\hline Preamble & $144 \mathrm{bits}$ & ACK rate & 1 Mbps \\
\hline
\end{tabular}

A two-state-Markov (On-Off) model is used to simulate voice sources with talkspurts. On and off states are modeled by random variables exponentially distributed with mean values $1.2 \mathrm{~s}$ and $1.8 \mathrm{~s}$, respectively [13, 14]. During On periods, voice traffic is modeled by a CBR source at $64 \mathrm{kbps}$, with packets of 160 bytes, simulating Pulse Code Modulation (PCM) voice [1]. Each simulation run lasts for $400 \mathrm{~s}$ and the starting time of each source is uniformly distributed between 1 and $11 \mathrm{~s}$. 
The simulation model assumes that coding/decoding delay, packet generation delay, and queuing delay at the recipient are negligible. Moreover, all packets have a maximum lifetime of $250 \mathrm{~ms}$, beyond which a packet is considered lost. Thus, the packet that takes more than $250 \mathrm{~ms}$ to arrive is discarded by the recipient. We arbitrarily choose $250 \mathrm{~ms}$ as packet lifetime to consider a value between good interactivity (less than 150 ms) and no interactivity (more than $400 \mathrm{~ms}$ ). When the end-to-end delay is smaller than the maximum lifetime the packet is received within an acceptable delay. Otherwise the packet is discarded and it is considered in the packet loss statistic. For PCM encoding, delivery rate should never drop under a percentage of 95\% of all generated packets, to prevent significant loss of quality [2]. Therefore, we have defined a threshold loss rate of 5\% for voice flows as a QoS parameter. This QoS parameter means that 95\% of the packets must arrive before packet lifetime expiration. Indeed, it is a pessimist assumption that is related to the PCM encoding scheme, but we are considering the worst case. This parameter has been used to determine the number of voice sources allowed for each scenario. Loss rate figures present a 5\%-loss rate curve to help identification of the number of voice sources.

Simulations are presented in two parts. The first one deals with single hop networks in order to evaluate the effect of network load on voice transmission capacity. The second one aims to investigate the effect of load, mobility, and node density on voice transmission capacity on multihop networks. Simulations take into account loss rate, delay, jitter, and consecutive losses for voice traffic.

\section{III.1. Single hop networks}

This subsection presents results related to network load on ad hoc networks based on IEEE 802.11. In these simulations, we have chosen simple scenarios in which the routing effect is minimized. These scenarios are composed of 40 fixed nodes with transmission range of $250 \mathrm{~m}$ in a $150 \mathrm{~m} \times 150 \mathrm{~m}$ area, which means that packets do not need to be routed because nodes can directly communicate with each other. Therefore, medium access time is the only parameter that influences on the network capacity. We consider that packet retransmissions due to collisions or transmission errors are included in the medium access time. Moreover, in order to evaluate the effect of load, background traffic is modeled by five CBR sources sending packets of 500 bytes at $200 \mathrm{kbps}$ and $250 \mathrm{kbps}$, simulating medium and high load conditions, respectively.

The first set of simulations verifies the impact of the network load on the medium access time. Figures $1(\mathrm{a})$ and $1(\mathrm{~b})$ show the influence of the network load on the transmission capacity due to medium access delay. According to Figure 1(a), 12 and 9 are the maximum number of voice sources allowed for 1 and $1.25 \mathrm{Mbps}$. Considering these 5\%-loss rate voice transmission capacities (12 and 9 voice sources), jitter remains almost constant (Figure 1(b)), which means that jitter is the same for a loss rate value, independently from background traffic. The results also reveal that the increase of the network load by 25\% reflects a drop on the network capacity of 25\%, which proves that network load can reduce the voice transmission capacity. 


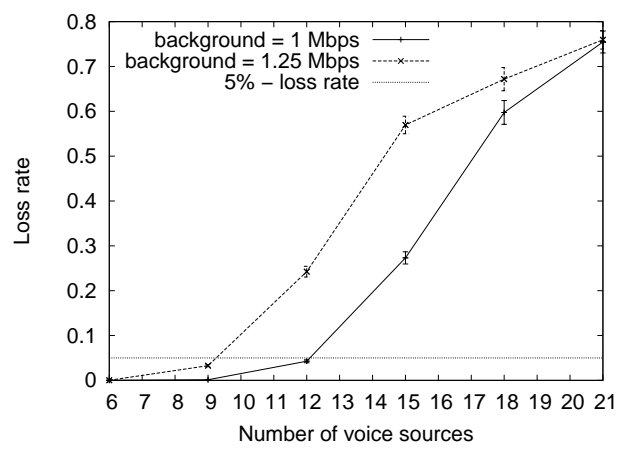

(a)

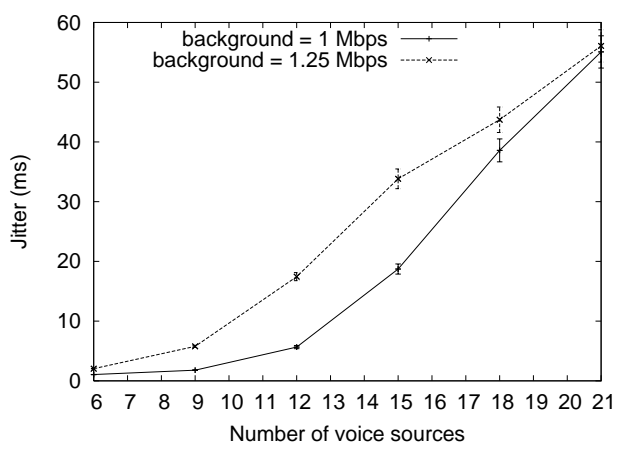

(b)

Figure 1: Effect of network load on voice transmission.

Figure $2(\mathrm{a})$ illustrates the CDF (Cumulative Distribution Function) of consecutive packet losses for 12 voice sources under a medium load and 9 voice sources under a high load. Although the two curves represent distinct scenarios, in both cases the maximum capacity of voice transmission is achieved. In Figure 2(a), the two curves behave almost the same. It occurs due to the fact that packet losses are mainly affected by the medium access mechanism, since it is a single hop communication and so there is no link failures or route discovery delays. As medium access time is influenced by the network load and by the number of voice sources, if we add these parameters in both scenarios, a similar total traffic is obtained, which leads to similar burst loss behaviors.

Figure 2 (b) presents the conditional PMF (Probability Mass Function) of the consecutive losses, given that a packet has been lost, as the following expression:

$$
P\left(k \mid 1^{s t}\right)=\frac{P(k) \bigcap P\left(1^{s t}\right)}{P\left(1^{s t}\right)}, k \in \mathrm{N},
$$

where $1^{\text {st }}$ means the event of losing a packet after a successful transmission or the first packet of a burst.

Figure 2(b) confirms the similarity in burst loss behavior as seen in Figure 2(a). Besides, it shows that the probability of up to 4 consecutive losses given that one has already occurred is greater than 80\%. This means that $80 \%$ of all burst losses are less or equal to 4 consecutive losses.

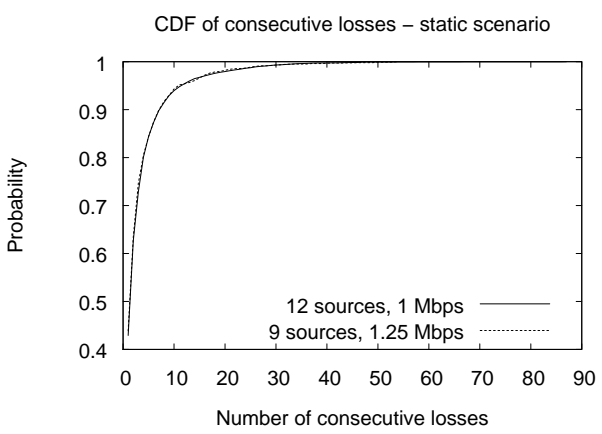

(a) CDF of consecutive losses

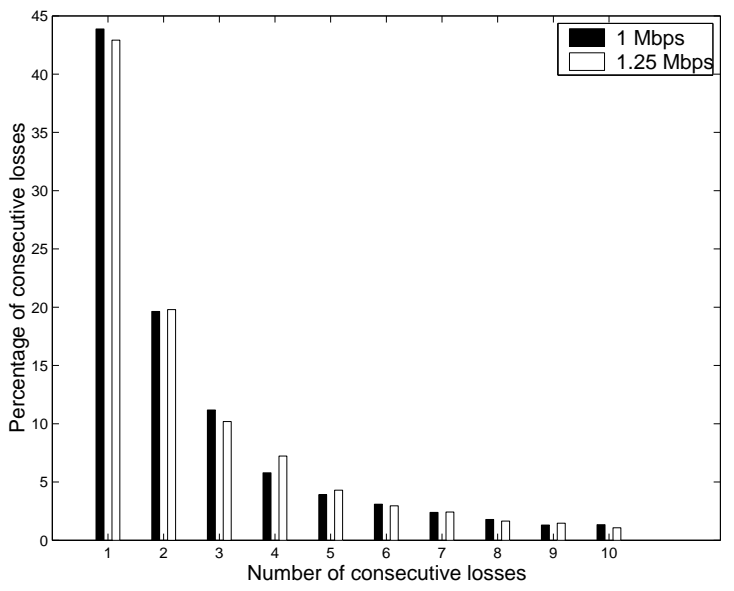

(b) Conditional PMF of consecutive losses

Figure 2: Effect of network load on consecutive losses. 
To summarize, results show that even when the network is loaded on single hop ad hoc networks, transmitting voice traffic is feasible. Nevertheless, increasing network load degrades network capacity since medium access time plays an important role in packet loss due to lifetime expiration. Concerning burst losses, as there are no route discovery procedures after the beginning of the simulation, main burst losses are small (less or equal to 4 consecutive losses).

\section{III.2. Multihop networks}

In this subsection, we present results related to the effect of mobility and node density on the capacity of voice transmission on multihop ad hoc networks. This kind of network is much more complex than single hop networks. In addition to the medium access problem, multihop networks have to cope with dynamic route discovery and lack of connectivity. Therefore, it is also important to assess voice transmission capacity on such networks. Performance evaluation of voice transmissions on multihop ad hoc networks has not been well explored yet.

Simulation scenarios consist of 40 nodes randomly placed in a $800 \mathrm{~m} \times 600 \mathrm{~m}$ area, which provide well connected scenarios with 1/12,000 $\mathrm{m}^{2}$ node density and transmission range of $250 \mathrm{~m}$. Source and destination are randomly picked among all nodes, one source and destination per node. We have defined two mobility levels: low and medium, with average speed $\left(s_{a}\right)$ of $1 \mathrm{~m} / \mathrm{s}$ and $4 \mathrm{~m} / \mathrm{s}$, respectively. In order to smooth node movements we have modified the random way point mobility model, which is a very popular and frequently used model [15, 16, 17]. In our model, the speed $s_{i}$ of a given node $i$ is uniformly distributed in the following interval: $0.8 s_{a} \leq s_{i} \leq 1.2 s_{a}$. We have simulated zero and low load conditions for both mobility levels. The high load scenario has not been used due to the low capacity of this kind of network, which means that it is not possible to transmit voice in this scenario. In these specific simulations, background traffic is modeled by 20 CBR sources at $16 \mathrm{kbps}$, instead of $5 \mathrm{CBR}$ sources as used on the single hop network experiments. In multihop networks, nodes might not interfere with each other, depending on their transmission range and position. Thus, according to the network topology, the selection of the source may affect the result. Therefore, we have increased the number of background traffic sources to balance the load and, consequently, to minimize the variance of our results.

Figures $3(\mathrm{a})$ and $3(\mathrm{~b})$ show the influence of mobility on network capacity according to the number of voice sources. With no load, we can have 10 voice sources transmitting simultaneously for low mobility and 4 voice sources for medium mobility (Figure 3(a)), while in a low loaded network with low mobility we can only have 5 voice sources (Figure 3(b)). It shows that mobility has a great impact on network capacity as a consequence of link failures and route discovery delay. Results related to medium mobility with low load are not plotted because in such scenario it is not possible to transmit voice flows with a loss rate below 5\%.
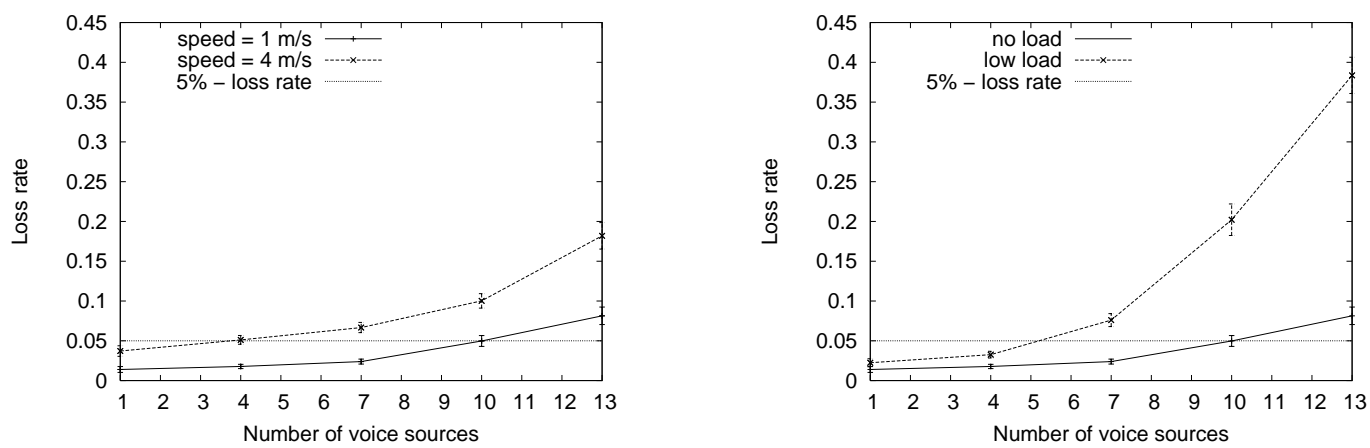


\section{Figure 3: Effect of mobility on loss rate.}

Figures 4(a) and 4(b) illustrate the influence of mobility on jitter, emphasizing the degradation of the capacity due to the increase of load and mobility. If we consider the voice transmission capacity (5\%-loss rate) from Figure 3(a), 10 voice sources at low mobility and 4 voice sources at medium mobility, we observe that jitter has increased more than 40\% (Figure 4(a)). When we fix the speed and vary the background traffic (Figure 4(b)), jitter remains almost constant, considering 10 voice sources transmitting under no load and 5 voice sources under low load (from Figure 3(b)). These results show that increasing background traffic increases medium access time, which leads to voice degradation. However, for the same loss rate, jitter remains constant because although medium access delay increases, the difference between consecutive delays does not change. This result is very similar to the one obtained for single hop networks when background traffic increases (Figure 1(b)). On the other hand, increasing mobility affects jitter in a different way due to link failures. Therefore, when considering mobility, for the same loss rate, jitter increases.

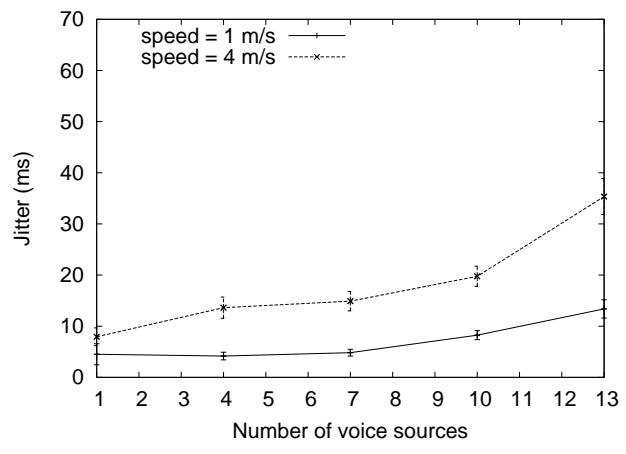

(a) No load

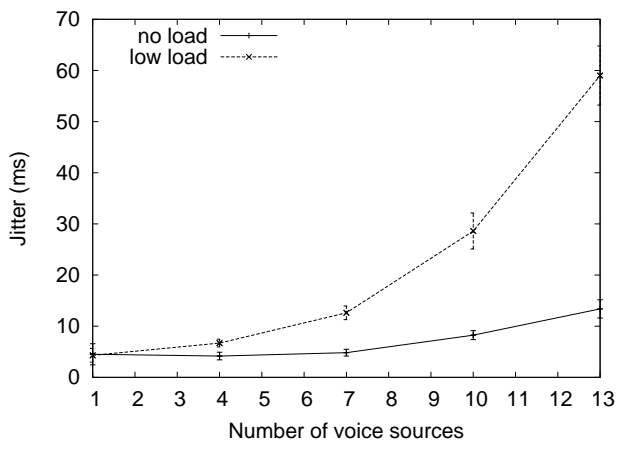

(b) Speed $=1 \mathrm{~m} / \mathrm{s}$

Figure 4: Effect of mobility on jitter.

Figure 5 presents the CDF of consecutive losses for a scenario with no load and average speed of $1 \mathrm{~m} / \mathrm{s}$. According to Figure 3(a), the capacity of this scenario is 10 voice sources and the loss rate for 7 and 13 voice sources is $2.4 \%$ and $8.1 \%$, respectively. Figure 5 shows that as the number of voice sources increases the CDF grows faster to one. It means that in a network with low traffic, for example 7 voice sources, larger burst losses are more significant due to link failures provoked by the mobility of the nodes, since for 7 voice sources the number of packets lost due to lifetime expiration is low. In other words, packet losses are mostly caused by routing discovery delay instead of congestion and medium access delay. Although the number of consecutive losses is plotted up to 250, it can achieve almost 2,000, while on a single hop network this number remains lower than 90 consecutive losses (Figure $2(\mathrm{a})$ ). 


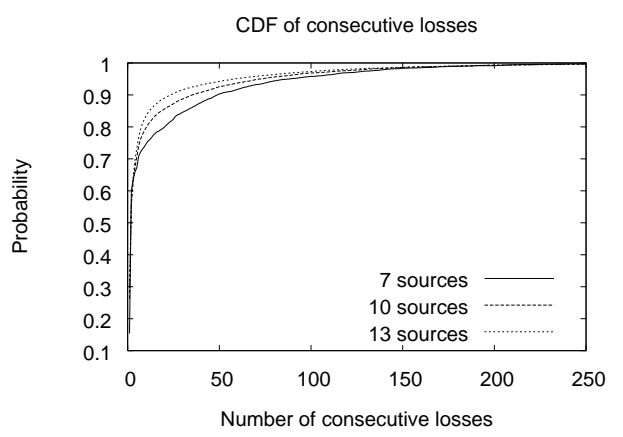

Figure 5: Consecutive losses $-1 \mathrm{~m} / \mathrm{s}$ and no load.

Figure $6(\mathrm{a})$ presents the conditional PMF of the consecutive losses, given that one packet loss has already occurred. It indicates that for a low loss rate (for instance, 7 voice sources) the probability of occurrence of one single packet loss is smaller, because most of the packet losses occur due to changes on topology. It also shows that as the number of voice sources increases the curve gets closer to the Pareto distribution, because it decays exponentially and it has a heavy tail.
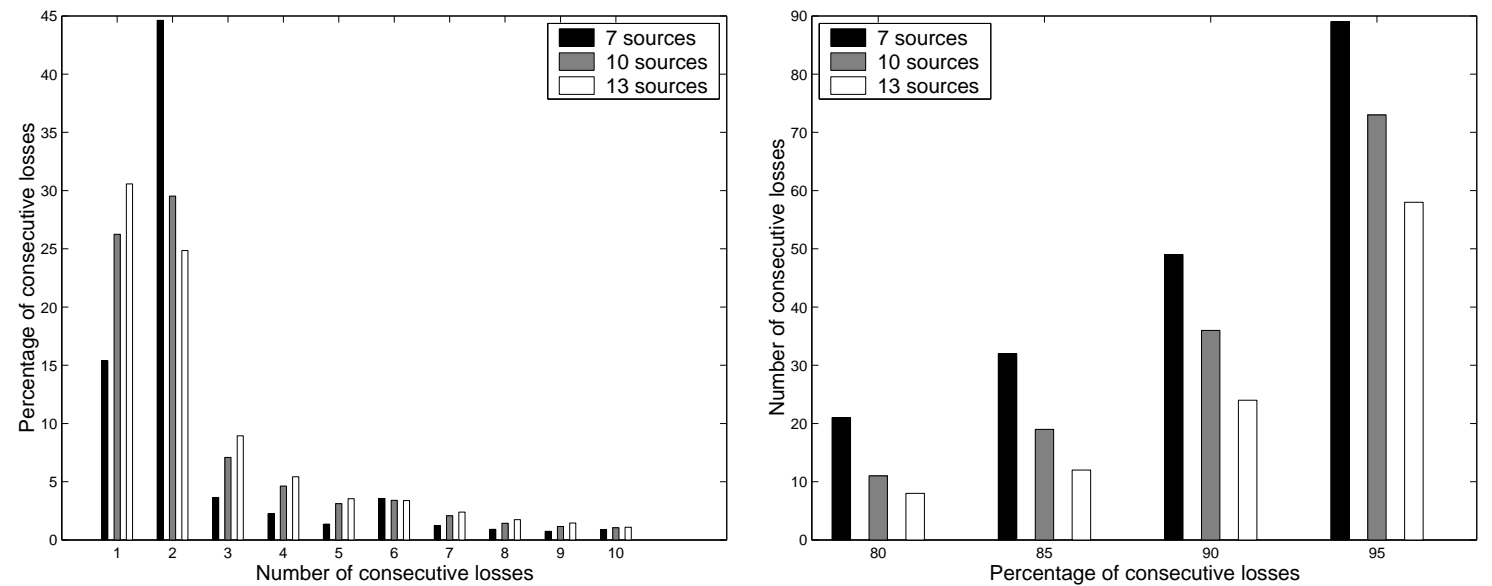

(a) Conditional PMF of consecutive losses

(b) Percentage of consecutive losses

\section{Figure 6: Consecutive loss $-1 \mathrm{~m} / \mathrm{s}$ and no load.}

Figure $6(\mathrm{~b})$ shows the difference even more clearly. It shows that for 10 voice sources $80 \%$ of the number of burst losses are less than 12 consecutive losses and only 5\% are more than 73 consecutive losses.

Figure 7 presents the CDF of the consecutive losses from scenarios with low mobility and medium mobility without background traffic. It can be noticed that the curve with low mobility grows faster than the other, proving that topology changes have a great impact on consecutive losses, but the difference between the scenarios with low mobility and medium mobility is small. 


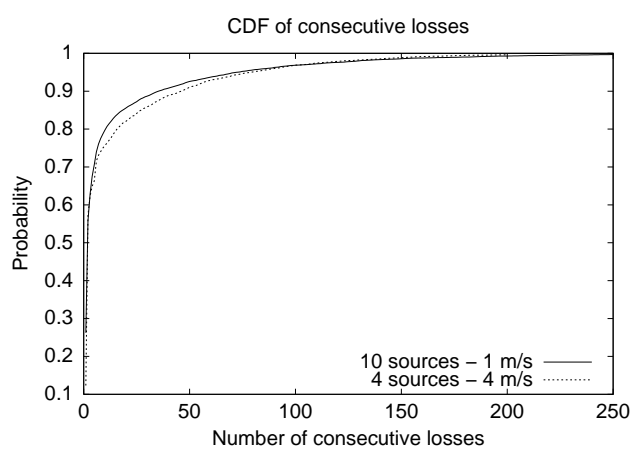

Figure 7: Effect of mobility on consecutive losses.

Figures 8 (a) and 8 (b) compare node mobility in scenarios without background traffic. From Figure $8(a)$ we can observe that a single packet loss and two consecutive packet losses correspond to more than 50\% of the packet losses and most of the losses occur up to 10 consecutive losses. Figure 8 (b) shows that $80 \%$ of the burst losses are less than 12 consecutive losses for low mobility and less than 17 for medium mobility.
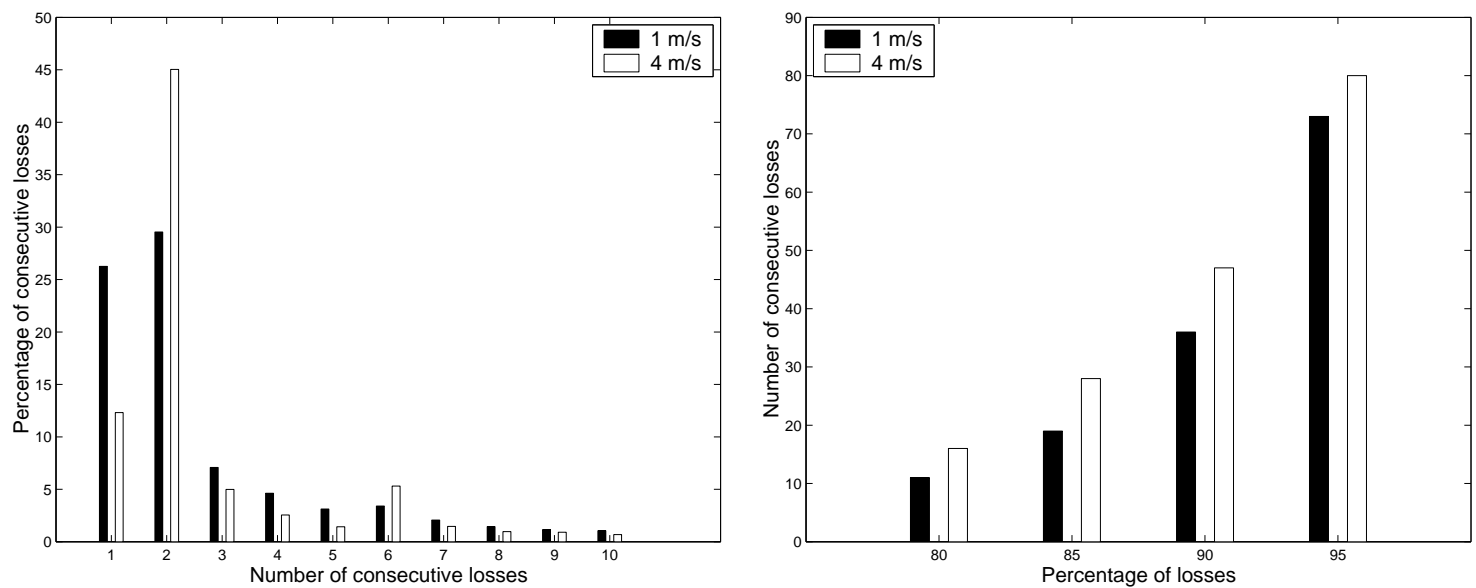

(a) Conditional PMF of consecutive losses

(b) Percentage of consecutive losses

Figure 8: Effect of mobility on consecutive losses.

We also address another important issue concerning the cause for packet losses. First, we have split lost packets in two groups according to the loss cause. The first group, named "lifetime", includes all packets dropped by the recipient due to lifetime expiration, previously defined as $250 \mathrm{~ms}$. The other group, named "others", contains packets lost for any other reason, such as collision, no route, that leads to MAC queue overflow.

Tables 3 and 4 summarize the influence of mobility and network load on the total number and on the percentage of losses. The increase of network load implies larger medium access time, which causes an increase in the number of packet losses due to lifetime expiration (see Total in Table 3). On the other hand, according to Table 4, as mobility increases the percentage of packet loss due to other reasons is larger, indicating that mobility has a greater impact on the second group than network load. This is expected because mobility reduces the packet delivery rate of routing protocols [15]. 
Table III: Loss cause with speed $1 \mathrm{~m} / \mathrm{s}$.

\begin{tabular}{|c|c|c|c|c|}
\cline { 2 - 5 } \multicolumn{1}{c|}{} & \multicolumn{4}{c|}{ Loss Cause } \\
\hline Load & Lifetime (\%) & Total & Others (\%) & Total \\
\hline zero & 69.14 & $3,315.3$ & 30.86 & 1,270 \\
\hline low & 68.43 & $14,434.3$ & 31.57 & $8,614.1$ \\
\hline
\end{tabular}

Table IV: Loss cause with no load.

\begin{tabular}{|c|c|c|c|c|}
\cline { 2 - 5 } \multicolumn{1}{c|}{} & \multicolumn{5}{c|}{ Loss Cause } \\
\hline Speed & Lifetime (\%) & Total & Others (\%) & Total \\
\hline $1 \mathrm{~m} / \mathrm{s}$ & 69.14 & $3,315.3$ & 30.86 & 1,270 \\
\hline $4 \mathrm{~m} / \mathrm{s}$ & 45.64 & $3,095.4$ & 54.36 & $3,638.5$ \\
\hline
\end{tabular}

The node density affects the network connectivity, which is strongly related to the network capacity of voice transmission. The following results refer to the effect of node density on multihop ad hoc networks. In order to analyze this effect, we have generated two other scenarios in which the node density has been changed by keeping the number of nodes constant and varying the simulation area. Thus, we have simulated three different areas. The first one is a $600 \mathrm{~m} \times 600 \mathrm{~m}$ field (small area), which represents a density of 1 node by $9,000 \mathrm{~m}^{2}$. The second one is an $800 \mathrm{~m} \times 600 \mathrm{~m}$ field (medium area) with a density of 1 node by $12,000 \mathrm{~m}^{2}$. The last one is a $1,200 \mathrm{~m} \times 500 \mathrm{~m}$ field (large area) with 1 node by $15,000 \mathrm{~m}^{2}$. The average speed is set to $4 \mathrm{~m} / \mathrm{s}$.

Both loss rate and jitter appear to have a similar behavior (Figure $9(\mathrm{a})$ and $9(\mathrm{~b})$ ). Due to the higher probability of link failures and the lack of connectivity, the large area cannot support voice traffic according to the QoS parameter of 95\% for packet delivery. The medium area performs better than the small area with a small number of voice sources, because of the effect of the medium access contention in the small area.

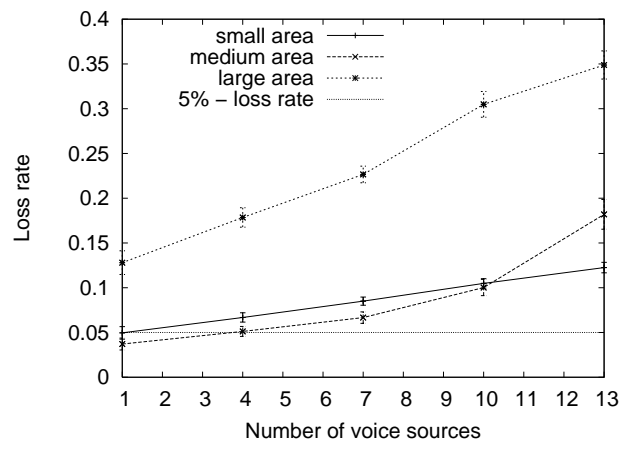

(a) Loss rate - no load

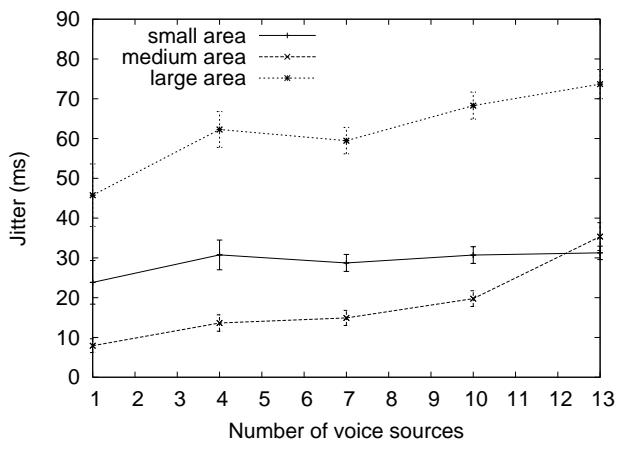

(b) Jitter - no load

Figure 9: Effect of node density on voice transmission.

Figure 10 shows that as the area decreases the CDF grows faster to one, which means that the network connectivity has direct influence on consecutive losses. Therefore, a network with a high level of connectivity and with a small maximum number of hops can reduce burst losses. 


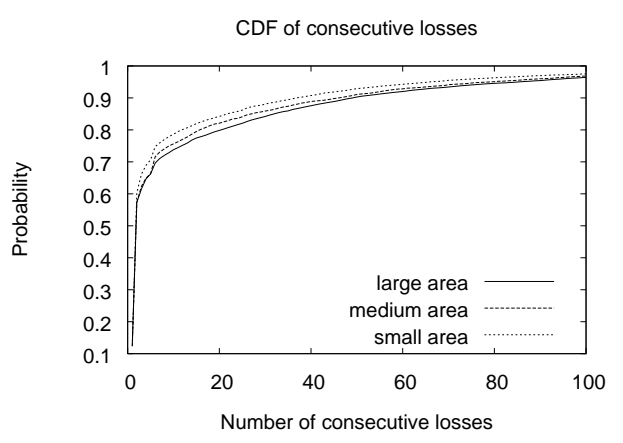

Figure 10: Effect of mobility on consecutive losses.

Results show that voice transmission capacity degrades with mobility since mobility leads to link breakages due to topology changes. These link breakages augment the length of burst losses, which achieved more than 2,000 packets. Routing discovery delay due to link failures is responsible for this behavior. Moreover, reducing node density has a negative effect over voice transmission capacity, due to the increase of link breakage probability and the increase of the number of hops needed to cover the higher distance between nodes. This effect is due to the way density has been reduced since simulation area has been increased instead of reducing the number of nodes.

\section{Conclusion}

This paper has analyzed voice transmission capacity on IEEE 802.11 ad hoc networks, more precisely, the influence of mobility on loss rate, delay, jitter, and consecutive losses. We have also addressed the impact of node density on voice transmission capacity. We have defined two Qos parameter thresholds to assess voice transmission capacity. The first one is related to interactivity and defines a $250 \mathrm{~ms}$ lifetime to each voice packet, beyond which the packet is considered lost. The second one is associated to voice integrity and defines a 5\%-maximum loss rate.

Results show that even in hostile scenarios, in which the network is loaded and nodes move constantly, transmitting voice is feasible, without losing interactivity. Nevertheless, the increase of mobility and network load degrades network capacity in different ways. Network load directly affects the medium access time causing packet losses due to lifetime expiration, while mobility affects other parameters related to routing, which enlarge packet losses.

Results reveal that changes in the network topology have a great impact on burst losses, since node density is strongly related to the network connectivity. Thus, high connectivity and a small number of hops reduce burst losses.

We have observed that consecutive losses are an important issue in ad hoc networks. In single hop networks the burst length achieved 90 packets while on multihop networks this value achieved more than 2,000 packets. Routing discovery delay due to link failures is the responsible for this discrepancy.

This work opens the road for some future novel research directions. New results might be obtained for other routing protocols. The use of mechanisms for providing service differentiation on ad hoc networks based on IEEE 802.11 might be explored in order to improve the capacity of voice transmission. Different codecs can be compared to G.711, but it is mandatory to select the loss rate parameter according to the codec. Estimating the loss rate that gives similar quality for each codec is not trivial. Bit-rate selection 
algorithms like ARF (Auto Rate Fallback) and RBAR (Received Based Auto Rate) can also be taken into account to verify the impact of different selections of bit rates, according to attenuation, noise, and other impairments. Another issue that can be addressed is developing a distributed medium access control for ad hoc networks to improve voice transmission capacity.

\section{Acknowledgments}

The authors would like to thank CNPq, CAPES, FAPERJ, COFECUB, and FUJB.

\section{References}

[1] KÖPSEL (A.), EBERT (J.-P.), WOLISZ (A.), A performance comparison of point and distributed coordination function of an IEEE 802.11 WLAN in the presence of real-time requirements, Workshop on Mobile Multimedia Communications (MoMuC2000), Tokyo, Japan, Oct. 2000.

[2] KÖPSEL (A.), WOLISZ (A.), Voice transmission in an IEEE 802.11 WLAN based access network, Workshop on Wireless Mobile Multimedia (WoWMoM'2001), Rome, Italy, July 2001.

[3] GARG (S.), KAPPES (M.), An experimental study of throughput for UDP and VoIP traffic in IEEE 802.11b networks, IEEE Wireless Communications and Networking Conference, pp. 1748-1753, Mar. 2003.

[4] HOLE (D.P.), TOBAGI (F.A.), Capacity of an IEEE 802.11b wireless LAN supporting VoIP, IEEE International Conference on Communications, pp. 196-201, June 2004 .

[5] DENG (J.), CHANG (R.S.), A priority scheme for IEEE 802.11 DCF access method, IEICE Transactions on Communications, E82-B, n 1, pp. 96-102, Jan. 1999.

[6] ARMENIA (S.), GALLUCCIO (L.), LEONARDI (A.), PALAZZO (S.), Transmission of VoIP traffic in multihop ad hoc IEEE 802.11b networks: Experimental results, Proceedings of the First International Conference on Wireless Internet, pp. 148-155, July 2005.

[7] Velloso (P.B.), RUBinstein (M.G.), DUARTE (O.C.M.B.), Analyzing voice transmission capacity on ad hoc networks, International conference on Communications Technology (ICCT 2003), Beijing, China, 2003.

[8] Velloso (P.B.), RUBinstein (M.G.), DUARTE (O.C.M.B.), The effect of mobility on voice transmission capacity in mobile ad hoc networks, The 3rd IEEE Latin American Network Operations and Management Symposium LANOMS'2003, Iguassu Falls, Brazil, Sept. 2003.

[9] ITU-T, One-way transmission time, Recommendation G.114, Mar. 1993.

[10] IEEE, IEEE standard 802.11, Wireless LAN medium access control (MAC) and physical layer (PHY) specifications, 1999.

[11] FALL (K.), VARADHAN (K.), The ns Manual, UC Berkeley, LBL, USC/ISI, and Xerox PARC, Oct. 2007.

[12] JOHNSON (D.), MALTZ (D.), BROCH (J.), DSR: The Dynamic Source Routing protocol for multihop wireless ad hoc networks, Ad Hoc Networking, ch. 5, pp. 139-172, Addison-Wesley, 2001.

[13] BRANDY (P.), A technique for investigating on/off patterns of speech, Bell Labs Tech Journal, 44, $\mathrm{n}^{\circ}$ 1, pp. 1-22, Jan. 1965.

[14] MARKOPOULOU (A.), TOBAGI (F.), KARAM (M.), Assessment of VoIP quality over internet backbones, IEEE Conference on Computer Communications (INFOCOM), New York, USA, June 2002. 
[15] DAS (S.R.), PERKINS (C.E.), ROYER (E.M.), Performance comparison of two on-demand routing protocols for ad hoc networks, IEEE Conference on Computer Communications (INFOCOM), Tel Aviv, Israel, pp. 3-12, Mar. 2000 .

[16] HOLLAND (G.), VAIDYA (N.H.), Analysis of TCP performance over mobile ad hoc networks, ACM Intern. Conf. on Mobile Computing and Networking (MobiCom 99), Seattle, USA, Aug. 1999.

[17] COSTA (L.H.M.K.), AMORIM (M.D.), FDIDA (S.), Reducing latency and overhead of route repair with controlled flooding, ACM/KIuwer Wireless Networks, 10, $\mathrm{n}^{\circ} 4$, pp. 347-358, July 2004 . 